\title{
DIAGNÓSTICO PRENATAL DE ACRETISMO PLACENTARIO: HALLAZGOS Y UTILIDAD DEL ULTRASONIDO Y LA RESONANCIA MAGNÉTICA. REPORTE DE CASOS EN EL HOSPITAL UNIVERSITARIO DE LA FUNDACIÓN SANTA FE DE BOGOTÁ (COLOMBIA)
}

Prenatal diagnosis of placental accretism: Findings and role of ultrasound and magnetic resonance. Cases report from university hospital of Fundación Santa Fe de Bogotá (Colombia)

Aleida Wilches-Llanos, $M D^{1}$; Gloria Palazuelos-Jiménez, $\mathrm{MD}^{2}$; Stephanie Trujillo-Calderón, $\mathrm{MD}^{3}$; Javier Romero-Enciso, $\mathrm{MD}, \mathrm{MSc}^{4}$

Recibido: Mayo 30/14 - Aceptado: Diciembre 15/14

\section{RESUMEN}

Objetivo: describir y revisar los hallazgos relevantes en imágenes diagnósticas del abordaje prenatal de pacientes con diagnóstico de acretismo placentario. Materiales y métodos: presentamos los casos de tres mujeres que fueron atendidas en un centro hospitalario de alta complejidad, con factores de riesgo para acretismo placentario y evaluación prenatal por ultrasonido (US) y resonancia magnética (RM), cuyo diagnóstico fue corroborado

1 Médico Radiólogo. Fellowship Imágenes de la Mujer. Departamento de Radiología e Imágenes Diagnósticas, Fundación Santa Fe de Bogotá (Colombia).

2 Médico Radiólogo, Centro de Imágenes de la Mujer. Departamento de Radiología e Imágenes Diagnósticas, Fundación Santa Fe de Bogotá (Colombia).

3 Médico, asistente de investigación, Departamento de Radiología e Imágenes Diagnósticas, Fundación Santa Fe de Bogotá (Colombia).

4 Médico Radiólogo. Director de Imágenes de la Mujer. Departamento de Radiología e Imágenes Diagnósticas, Fundación Santa Fe de Bogotá. Profesor de cátedra, Facultad de Medicina, Universidad de los Andes, Bogotá (Colombia).javieraromero@yahoo.com. con estudio anatomopatológico. Se describen los aspectos clínicos, los hallazgos por imágenes y su correlación histológica. Se realizó una búsqueda de la literatura a través de PubMed, Lilacs y EBSCO, se incluyeron artículos originales, revisiones de tema y reportes de casos sobre la utilidad y los aspectos más importantes del US y la RM en el diagnóstico prenatal de acretismo placentario.

Resultados: se incluyeron 11 artículos originales, 8 de revisión y 1 reporte caso. La placenta previa asociada a lagunas placentarias en ultrasonido y en RM a bandas hipointensas placentarias y el abombamiento del útero son considerados como los principales signos de acretismo. El US asociado a Doppler color tiene una sensibilidad de $97 \%$ y una especificidad del $92 \%$. La RM puede definir el compromiso uterino y de los órganos vecinos, con una sensibilidad que varía entre el 77 y el 89,6\%, la especificidad es cercana al $92 \%$.

Conclusiones: el abordaje prenatal precoz a través de las imágenes diagnósticas no invasivas brinda 
información de utilidad a los clínicos del compromiso y la extensión en la evaluación de pacientes con factores de riesgo para acretismo placentario.

Palabras clave: placenta acreta, placenta, ultrasonografía, imagen por resonancia magnética, diagnóstico prenatal.

\section{ABSTRACT}

Objective: To describe and review the relevant findings of the prenatal diagnostic imaging approach in patients placenta accreta diagnosis.

Materials and methods: We present 3 cases of women with risk factors for placenta accreta assessed prenatally at a high-complexity hospital, using ultrasound (US) and magnetic resonance imaging (MRI), with histopathological confirmation of the diagnosis. Clinical and imaging findings are described, together with their histological correlation. A search of the literature was conducted through PubMed, LILACS, and Ebsco, including original papers, topic reviews and case reports on the use and more important aspects of US and MRI in the pre-natal diagnosis of placenta accreta.

Results: 11 Original articles, 8 Review articles and 1 case report from our country were included. Placenta accreta is an obstetric pathology that threatens the health of the mother and fetus. Placenta previa associated to placental gaps in US and intraplacental hypointense bands and uterine bulge in MRI are considered as the major signs of accretism. US with color Doppler has $97 \%$ of sensitivity and $92 \%$ of specificity, MRI defines uterine and neighboring organs compromise $(\mathrm{s}=$ $77-89.6 \%, \mathrm{e}=92 \%$ ).

Conclusion: Early prenatal approach through noninvasive diagnostic imaging plays a usefull role providing information about the compromise and extention in patients with risk factors for placental accretism.

Key words: Placenta Accreta, Placenta, Ultrasonography, Magnetic Resonance Imaging, prenatal diagnosis.

\section{INTRODUCCIÓN}

El acretismo placentario (AP) es una patología obstétrica con alta morbimortalidad materno-fetal $(9,5 \%)(1,2)$, que se presenta en 1:2500 nacimientos, con una incidencia de $0,9 \%$ (3). Se define como la inserción anormal de la placenta secundaria a un defecto de la decidua basal, que permite que las vellosidades coriónicas invadan el miometrio (4). De acuerdo con la profundidad se divide en: acreta si existe compromiso de la capa superficial, increta si invade el miometrio y percreta si invade la serosa pudiendo afectar los tejidos adyacentes (5). Por tanto, se ha descrito que el manejo de la placenta acreta puede acompañarse, hasta un $13 \%$, de lesiones en la vía urinaria, intestino o rotura uterina (6). Entre los factores de riesgo se encuentran: antecedente de cesárea previa (a mayor número de cesáreas mayor es el riesgo), placenta previa (PP), fertilización in vitro (FIV), edad materna mayor de 35 años y cirugías uterinas previas $(5,7,8)$. La histerectomía puede conducir a complicaciones por lesión de órganos adyacentes y a comorbilidades graves (cistostomía, UCI, etc.) $(9,10)$. El diagnóstico se confirma por la ausencia de la decidua y la presencia de vellosidades placentarias en el miometrio, ya sea en los especímenes de histerectomía o en placas con muestras de placenta (11); sin embargo, en algunos casos la hemorragia puede ser controlada sin histerectomía, por lo que el análisis patológico no está disponible (figuras 1C, 2C, 3C) $(6,12)$. Un diagnóstico prenatal precoz y preciso permite evaluar las diferentes alternativas terapéuticas con el fin de disminuir la morbimortalidad asociada $(6,13)$.

Las imágenes diagnósticas son importantes en la evaluación prenatal de pacientes con factores de riesgo y brindan a los clínicos información relevante sobre la extensión y el manejo. Actualmente, en pacientes con sospecha de AP se recomienda realizar primero el ultrasonido (US) transabdominal en escala de grises y con Doppler color, seguido por la resonancia magnética (RM) de pelvis en los casos en los que el ultrasonido no es concluyente o la evaluación de la placenta es limitada (14). 

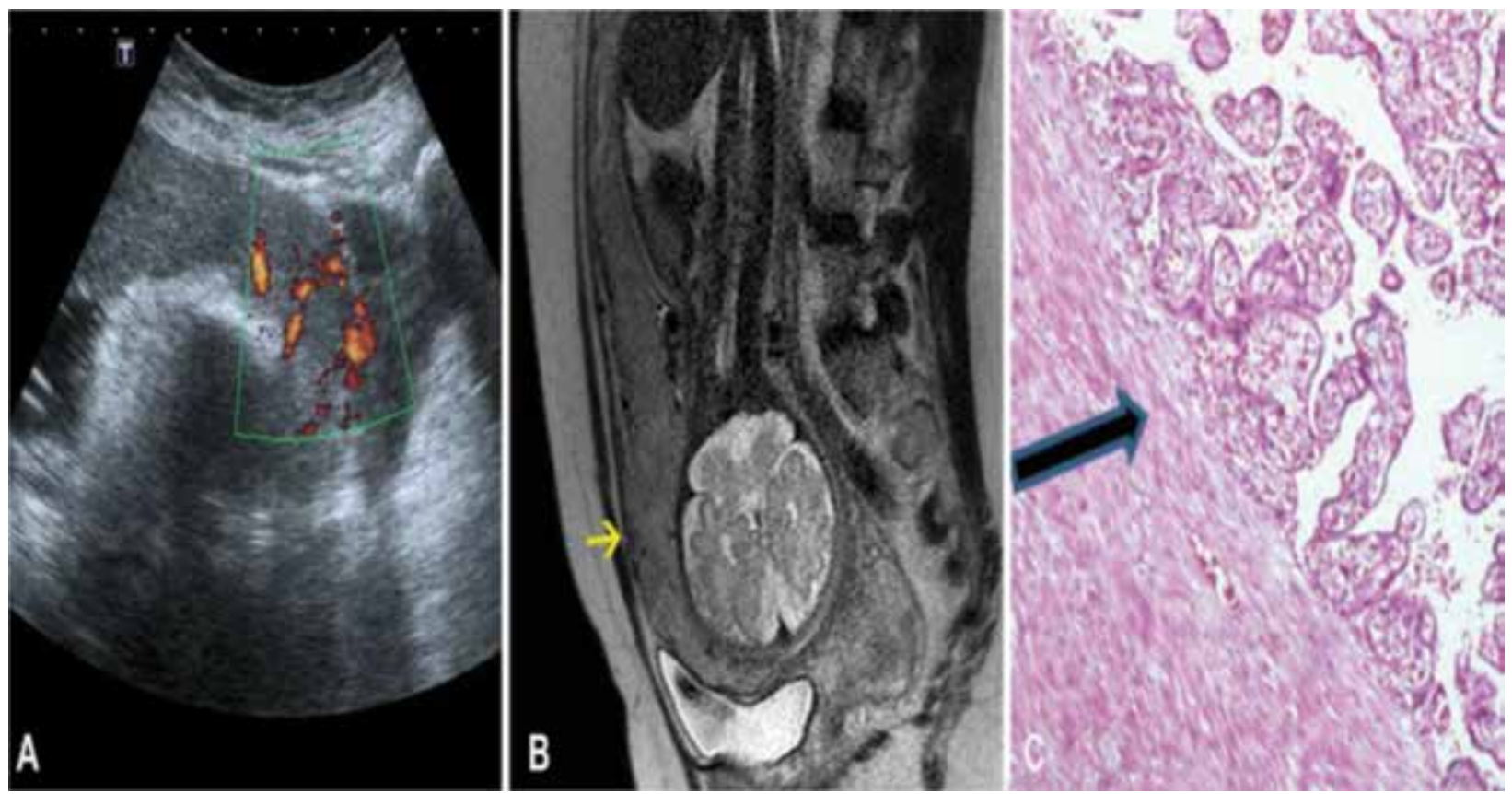

Figura 1. A) US - Pérdida del plano de separación vejiga-placenta. El Doppler color muestra vasos placentarios que se extienden a la pared vesical. B) RM - T2, sagital. Placenta corporal anterior de inserción baja, abombamiento uterino y pérdida de la línea hipointensa entre miometrio y placenta. C) Evaluación microscópica: vellosidades en contacto con el miometrio (flecha negra) sin interposición de decidua.

Debido a que la incidencia del AP ha venido en aumento, el reto de los clínicos es identificar pacientes con factores de riesgo, realizar una evaluación prenatal precoz y determinar el manejo adecuado, con el fin de disminuir las tasas de morbimortalidad materno-fetal asociadas. De esta manera, el uso de imágenes diagnósticas no invasivas puede ser un pilar fundamental en el abordaje. El objetivo de este estudio es presentar tres casos de acretismo placentario, describir los hallazgos normales de la placenta y las imágenes de la placenta acreta en el ultrasonido y la RM, y hacer una revisión del desempeño operativo de estas pruebas diagnósticas en esta entidad.

\section{REPORTE DE CASOS}

Se describen tres casos de acretismo placentario atendidos entre 2009 y 2014 en el Hospital Universitario de la Fundación Santa Fe, centro privado de alta complejidad ubicado en Bogotá, que atiende pacientes del aseguramiento privado y contributivo en el sistema de seguridad social en Colombia.
Caso 1. Paciente de 37 años, G3P1A1C1V1, embarazo de 37 semanas. El US evidenció placenta previa y pérdida del plano de separación con la pared uterina y la vejiga. En RM se observa placenta con señal de intensidad heterogénea, segmento delgado, ausencia focal de línea hipointensa retroplacentaria y lobulación del contorno uterino. En la cesárea se evidenció serosa infiltrada por vasos placentarios sin comprometer órganos pélvicos; se realizó histerectomía sin complicaciones. El examen anatomopatológico mostró placenta increta del $90 \%$ en tercio inferior e istmo y áreas de implantación con vellosidades en contacto con el miometrio sin interposición de decidua. Adecuada evolución (figuras 1A, 1B y 1C).

Caso 2. Paciente de 35 años, G2P1C1V1, embarazo de 31,3 semanas, tricorial, triamniótico, producto de fertilización in vitro (FIV). Placentas 1 y 3 anteriores previas y lagunas con flujo turbulento en US, placenta 2 anterior derecha. Lobulación e intensidad de señal heterogénea en RM. Serosa uterina infiltrada por vellosidades coriales; durante 
cesárea se realizó histerectomía sin complicaciones. Se realizó estudio anatomopatológico con solución de continuidad de $5 \times 7 \mathrm{~cm}$ en cara anterior con salida de tejido placentario e infiltración al miometrio. Microscópicamente se evidenciaron focos de acretismo, vellosidades maduras con áreas de fusión e hiperplasia de nudos sincitiales. Adecuada evolución (figuras 2A, 2B y 2C).

Caso 3. Paciente de 39 años, G4P1A2C1, embarazo de 33 semanas, gemelar, producto de FIV. En US se observó placenta 1 posterior oclusiva total que no permitía descartar acretismo, y placenta 2 anterior; Doppler con lagunas venosas con flujo turbulento. Lobulación del contorno del útero asociado a pérdida focal de la línea hipointensa retroplacentaria en RM. La cesárea evidenció vejiga adherida al segmento uterino, várices en repliegue vésico-uterino y segmento. Se realizó histerectomía.
Presentó comorbilidades dadas por transfusión de hemoderivados, cistostomía por lesión vesical e ingreso a UCI, posteriormente reintervención con ligadura de arterias hipogástricas. Fue dada de alta por adecuada evolución 15 días después. En el estudio macroscópico se observó placenta firmemente adherida a la superficie endometrial y miometral a $1 \mathrm{~cm}$ de la serosa, ocupando la totalidad del orificio cervical interno, microscópicamente se evidencian focos de acretismo (figuras 3A, 3B y 3C).

\section{MATERIALES Y MÉTODOS}

Con las palabras clave "Ultrasonography", "Placenta Accreta" y "Magnetic Resonance Imaging", se realizó una búsqueda en Medline vía PubMed, SciELO y EBSCO de artículos originales, revisiones de tema y reportes de casos publicados en inglés y
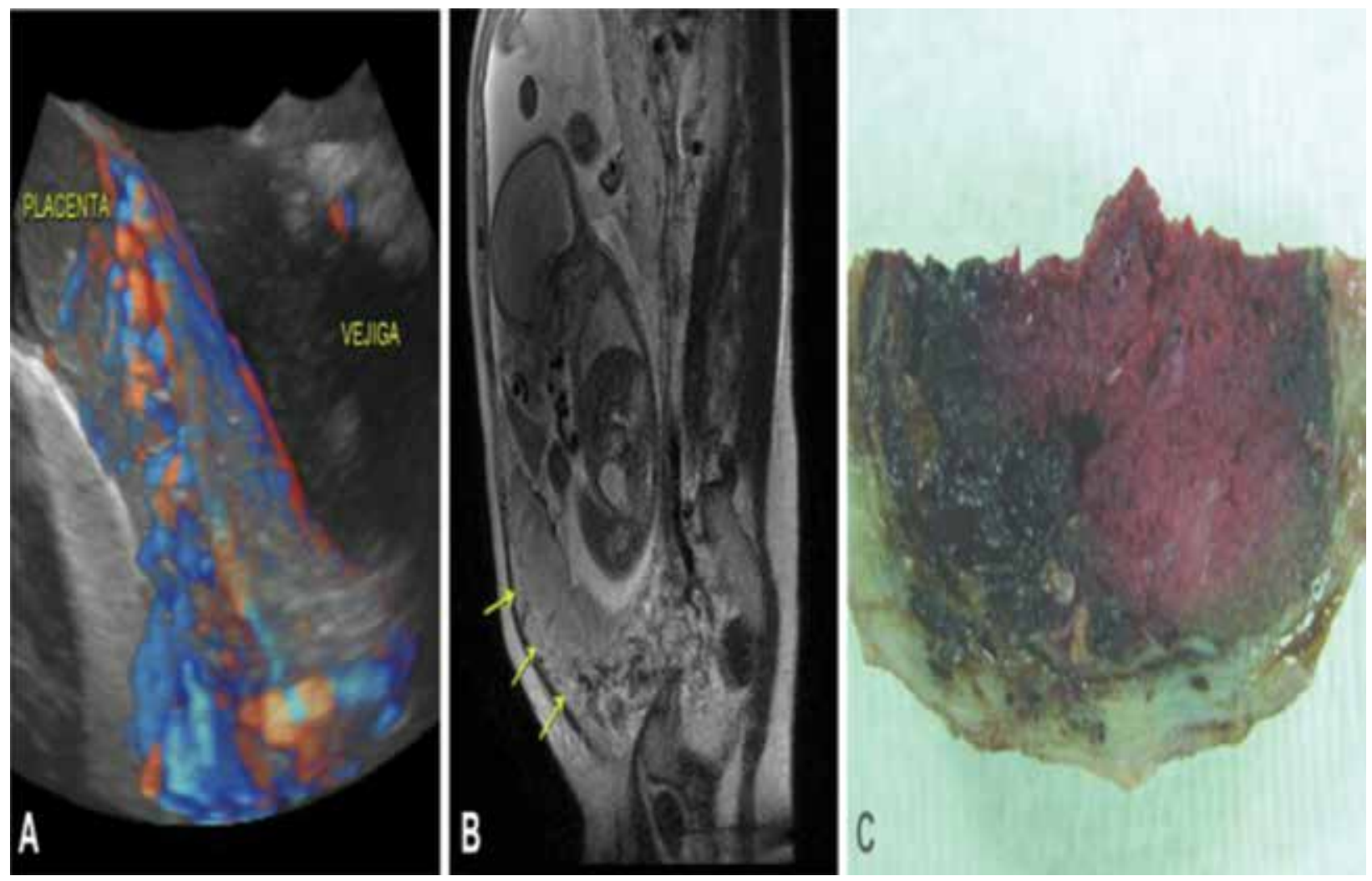

Figura 2. A) US transvaginal, Doppler color. Placenta previa, lagunas venosas con flujo turbulento, pobre diferenciación entre placenta y miometrio. B) RM T2, plano sagital. Pérdida de la línea hipointensa retroplacentaria, banda hipointensa en contacto con el miometrio. C) Estudio anatomopatológico, imagen macroscópica. Infiltración placentaria al miometrio. 

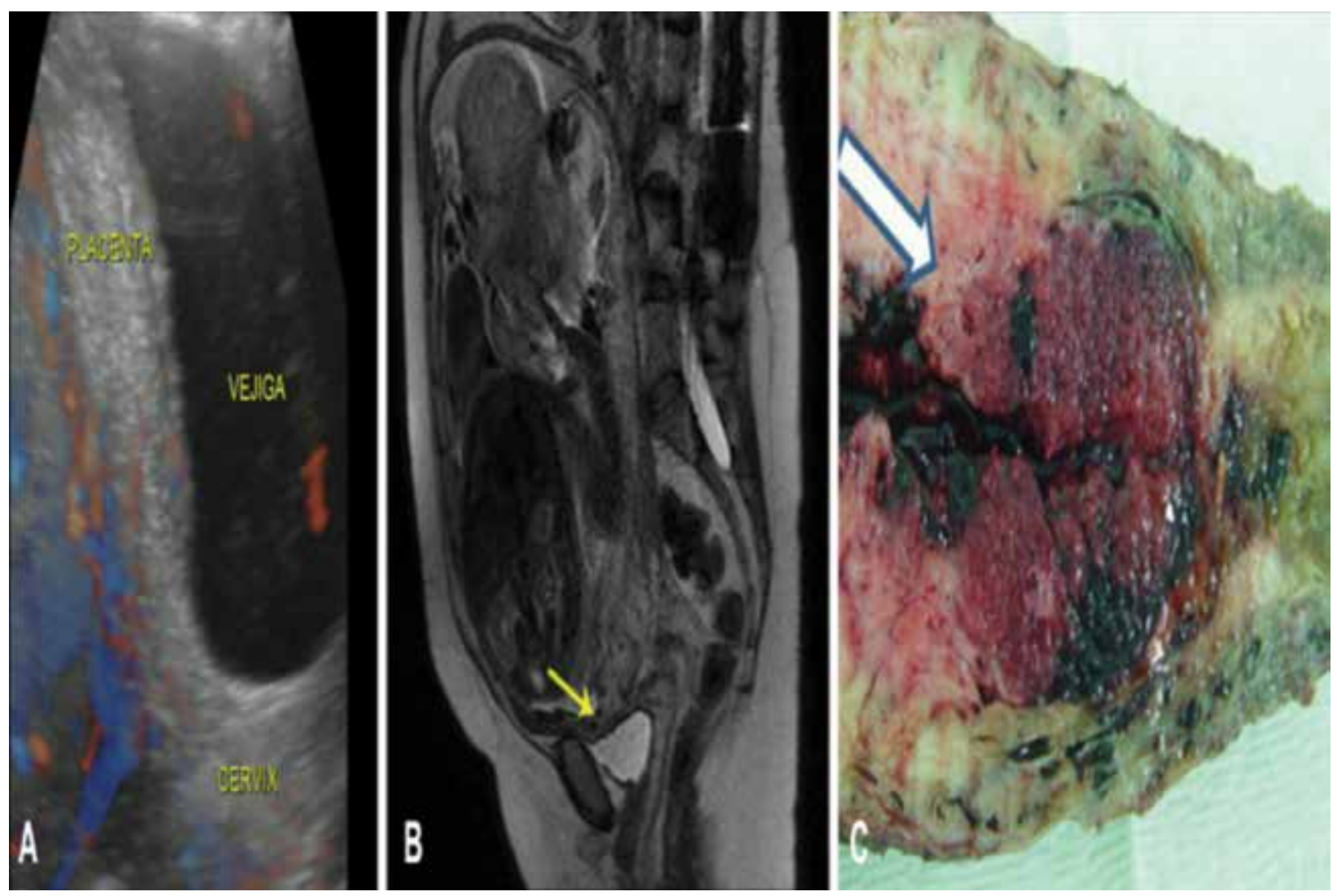

Figura 3. A) US transvaginal, Doppler color. Placenta previa posterior, lagunas venosas con flujo turbulento, vasos placentarios en miometrio. B) RM, sagital, T2 Fiesta (Fast Imaging Employing Steady State Acquisition- GE®). Pérdida de la línea posterior, tejido placentario en contacto con la vejiga e imágenes lineales con ausencia de señal (vasos placentarios). C) Estudio anatomopatológico, imagen macroscópica. Segmento y canal endocervical con placenta de implantación previa y firmemente adherida al miometrio (flecha blanca).

español, que describieran las características clínicas y el diagnóstico del acretismo placentario.

Consideraciones éticas. Se solicitó a las pacientes autorización para revisar los datos en la historia clínica y la publicación de las imágenes. Se garantizó la confidencialidad de la información protegiendo los derechos de las pacientes.

\section{RESULTADOS}

La búsqueda arrojó un total de 53 artículos. Se incluyeron 13 artículos originales, 8 de revisión y 1 reporte de casos de nuestro país.

Hallazgos normales de la placenta con el ultrasonido. En el segundo trimestre la placenta es homogéneamente ecogénica, con algunos septos delgados, adyacente a un miometrio hipoecogénico (3); hacia el tercer trimestre aparecen calcificaciones y lagos venosos con flujo laminar, dándole una apariencia heterogénea $(3,14)$. La línea retroplacentaria (desidua) es regular e hipoecoica y presenta flujo sanguíneo de distribución paralela (11).

Hallazgos normales con la RM. En las RM las secuencias utilizadas son FSE, T2 y T2 Fiesta (Fast Imaging Employing Steady State Acquisition-GE@) $(4,15)$, por lo menos una secuencia potenciada en T1 se adquiere para detectar zonas de hemorragia subcorial (15). En las secuencias ponderadas T2 la placenta presenta señal homogénea en las semanas 19 a 23 en el $85 \%$ de los casos, dato que excluye el diagnóstico de acretismo. El 90,7\% de las placentas se presentan ligeramente lobuladas en las semanas 24-31; el grado de lobulación y heterogeneidad 
aumenta con la edad gestacional (11). En la placenta normal pueden verse septos delgados de baja señal entre los lobulillos (16). El miometrio en T2 se presenta como una capa vascular de alta señal de intensidad intercalada entre dos capas finas de baja señal de intensidad dándole una apariencia trilaminar; sin embargo, también puede visualizarse de menor grosor sin que sugiera placentación anormal $(11,15)$.

Hallazgos de acretismo placentario (AP) con el ultrasonido y la $R M$. En pacientes con alto riesgo de AP se sugiere evaluar el miometrio anterior y las paredes vesicales en las semanas 18 a 20 (17). Las características ecográficas del AP incluyen: lagunas placentarias que se continúan al miometrio (figuras 2A y 3A), pérdida del espacio normal claro retroplacentario y disminución o interrupción de la interfase hiperecogénica de la serosa uterina con la pared vesical (3). La placenta previa y las lagunas placentarias son los hallazgos más importantes (17); las lagunas placentarias corresponden a estructuras vasculares dentro del parénquima dándole un aspecto de "queso suizo", y son el hallazgo ecográfico con mayor valor predictivo positivo (VPP) (92\%); a mayor número de lagunas aumenta el riesgo $(3,17)$. Debido a que el $88 \%$ de las placentas previas están asociadas a algún grado acretismo, se recomienda realizar una evaluación con Doppler color; en AP se pueden observar vasos placentarios que penetran el miometrio representando el sitio de la invasión (figura 1A) (10, 11). La ausencia del espacio claro retroplacentario tiene baja sensibilidad (7\%), ya que puede estar ausente en embarazos normales (13). La pérdida de la visualización del miometrio se presenta como un engrosamiento menor de $1 \mathrm{~mm}$ (18). El adelgazamiento o la interrupción de la interfase hiperecogénica de la serosa con la vejiga presenta una baja sensibilidad (18\%), pero una especificidad del $100 \%$ (19). La invasión de la pared vesical se presenta como irregularidad con una amplia vascularización (11).

La resonancia magnética (RM) puede definir el compromiso uterino y de los órganos vecinos
(11, 20). Los hallazgos específicos en RM son: abombamiento del útero, placenta de intensidad de señal heterogénea y bandas gruesas intraplacentarias hipointensas en las imágenes potenciadas en T2 $(11,15)$. La irregularidad o interrupción de la hipointensidad normal de la pared vesical y el aspecto nodular del contorno de la vejiga pueden ser hallazgos en la invasión vesical $(11,21)$. El abombamiento del útero es el signo más útil, y aunque no está claramente definido, se ha descrito como el cambio de la forma de pera invertida normal del útero grávido dándole aspecto de "reloj de arena”, también se describe como un contorno convexo en el borde placentario (figura 1B) (21). Las bandas intraplacentarias hipointensas en T2 son más gruesas que los tabiques de la placenta normal y se extienden dentro de la placenta desde la interfase placenta-miometrio (figura 2B y 3B) (11). La interrupción de la línea hipointensa retroplacentaria representa zonas de invasión de vellosidades coriales; sin embargo, sin otros signos asociados es un pobre predictor de AP (15).

Rendimiento diagnóstico. Meng y Song (22), en una revisión sistemática de 13 estudios, informan para el ultrasonido una sensibilidad del $83 \%$ (IC $95 \%$ : 77-88) con una especificidad del $95 \%$ (IC $95 \%$ : 93-96). Para la RM informan una sensibilidad del $82 \%$ (IC $95 \%$ : 72-90) y una especificidad del $88 \%$ (IC $95 \%$ : 81-94).

\section{CONCLUSIONES}

El abordaje prenatal precoz a través de las imágenes diagnósticas no invasivas brinda información de utilidad sobre el compromiso y la extensión en la evaluación de pacientes con factores de riesgo para acretismo placentario. La placenta previa asociada a lagunas placentarias en ultrasonido y en RM a bandas hipointensas placentarias y abombamiento del útero son considerados como los principales signos de acretismo. La RM debe reservarse a casos con hallazgos ecográficos equívocos o para evaluar zonas del útero de difícil acceso en US, como la placenta posterior. 


\section{AGRADECIMIENTOS}

Agradecemos a las doctoras Catalina Buriticá y Diana Palacios, del Departamento de Patología de la Fundación Santa Fe de Bogotá.

\section{REFERENCIAS}

1. Miller DA, Chollet JA, Goodwin TM. Clinical risk factors for placenta previa-placenta accreta. Am J Obstet Gynecol. 1997;177:210-4.

2. Fox H. Placenta Accreta, 1945-1969. Obstet Gynecol Surv. 1972;27:475-90.

3. Riteau AS, Tassin M, Chambon G, Vaillant C, de Laveaucoupet J, Quéré MP, et al. Accuracy of Ultrasonography and Magnetic Resonance Imaging in the Diagnosis of Placenta Accreta. PLoS One. 2014;9:e94866.

4. Palacios Jaraquemada JM, Bruno CH. Magnetic resonance imaging in 300 cases of placenta accreta: surgical correlation of new findings. Acta Obstet Gynecol Scand. 2005;84:716-24.

5. Wortman AC, Alexander JM. Placenta Accreta, Increta, and Percreta. Obstet Gynecol Clin North Am. 2013;40:137-54.

6. Fitzpatrick KE, Sellers S, Spark P, Kurinczuk JJ, Brocklehurst P, Knight M. The management and outcomes of placenta accreta, increta, and percreta in the UK: a population-based descriptive study. BJOG. 2014;121:62-70.

7. Wu S, Kocherginsky M, Hibbard JU. Abnormal placentation: twenty-year analysis. Am J Obstet Gynecol 2005;192:1458-61.

8. Esh-Broder E, Ariel I, Abas-Bashir N, Bdolah Y, Celnikier DH. Placenta accreta is associated with IVF pregnancies: a retrospective chart review. BJOG. 2011;118:1084-9.

9. Varghese B, Singh N, George RA. Magnetic resonance imaging of placenta accreta. Indian J Radiol Imaging. 2013;23:379-85.

10. Usta IM, Hobeika EM, Musa AA, Gabriel GE. Placenta previa-accreta: risk factors and complications. Am J Obstet Gynecol. 2005;193:1045-9.

11. Warshak CR, Eskander R, Hull AD, Scioscia AL, Mattrey RF, Benirschke K, et al. Accuracy of ultrasonography and magnetic resonance imaging in the diagnosis of placenta accreta. Obstet Gynecol. 2006;108:573-81.

12. Ferreira FE, Angulo M. Predicción del acretismo placentario con ultrasonido Doppler en el Hospital Universitario de Neiva, estudio de cohorte (Spanish). Rev Colomb Obstet Ginecol. 2007;58:290-5.

13. Comstock $\mathrm{CH}$. Antenatal diagnosis of placenta accreta: a review. Ultrasound Obstet Gynecol. 2005;26:89-96.

14. Derman AY, Haberman S, Zelenko N, Opsha O, Flyer M. MRI of Placenta Accreta: A New Imaging Perspective. AJR Am J Roentgenol. 2011;197:1514-21.

15. Kim JA. Magnetic resonance imaging with true fast imaging with steady-state precession and halfFourier acquisition single-shot turbo spin-echo sequences in cases of suspected placenta accreta. Acta radiologica (Stockholm, Sweden: 1987). 2004;45:692-8.

16. Blaicher W, Brugger PC, Mittermayer C, Schwindt J, Deutinger J, Bernaschek G, et al. Magnetic resonance imaging of the normal placenta. Eur J Radiol. 2006;57:256-60.

17. Baughman WC, Corteville JE, Shah RR. Placenta Accreta: Spectrum of US and MR Imaging Findings. RadioGraphics. 2008;28:1905-16.

18. Twickler DM, Balis AB, Santos R, Martin L, Malone $\mathrm{S}$, et al. Color flow mapping for myometrial invasion in women with a prior cesarean delivery. J Matern Fetal Med. 2000;9:330-5.

19. Shih JC, Palacios JM, Su YN, Shyu MK, Lin CH, Lin SY, et al. Role of three-dimensional power Doppler in the antenatal diagnosis of placenta accreta: comparison with gray-scale and color Doppler techniques. Ultrasound Obstet Gynecol. 2009;33:193-203.

20. Elhawary TM, Dabees NL, Youssef MA. Diagnostic value of ultrasonography and magnetic resonance imaging in pregnant women at risk for placenta accreta. J Matern Fetal Neonatal Med. 2013;26:1443-9. 
21. Yang JI, Lim YK, Kim HS, Chang KH, Lee JP, Ryu HS. Sonographic findings of placental lacunae and the prediction of adherent placenta in women with placenta previa totalis and prior Cesarean section. Ultrasound Obstet Gynecol. 2006;28:178-82.
22. Meng X, Xie L, Song W. Comparing the diagnostic value of ultrasound and magnetic resonance imaging for placenta accreta: a systematic review and meta-analysis. Ultrasound in medicine \& biology. 2013;39:1958-65. 